\title{
Comparative pathogenesis of Ebola virus and Reston virus infection in humanized mice
}

\author{
Beatriz Escudero-Pérez, ${ }^{1,2}$ Paula Ruibal, ${ }^{1}$ Monika Rottstegge, ${ }^{1,2}$ Anja Lüdtke, ${ }^{1}$ Julia R. Port, ${ }^{1,2}$ \\ Kristin Hartmann, ${ }^{3}$ Sergio Gómez-Medina, ${ }^{1,2}$ Jürgen Müller-Guhl,, ${ }^{1,4}$ Emily V. Nelson,, \\ Susanne Krasemann, ${ }^{3}$ Estefanía Rodríguez, ${ }^{4}$ and César Muñoz-Fontela ${ }^{1,2}$ \\ 'Bernhard Nocht Institute for Tropical Medicine, Hamburg, Germany. ${ }^{2}$ German Center for Infection Research (DZIF), \\ Partner Site Hamburg, Hamburg, Germany. ${ }^{3}$ Institute for Neuropathology, University Medical Center Hamburg-Eppendorf, \\ Hamburg, Germany. ${ }^{4}$ Heinrich Pette Institute, Leibniz Institute for Experimental Virology, Hamburg, Germany.
}

Filoviruses of the genus Ebolavirus include 6 species with marked differences in their ability to cause disease in humans. From the highly virulent Ebola virus to the seemingly nonpathogenic Reston virus, case fatality rates can range between $0 \%$ and $90 \%$. In order to understand the molecular basis of these differences, it is imperative to establish disease models that recapitulate human disease as faithfully as possible. Nonhuman primates (NHPs) are the gold-standard models for filovirus pathogenesis, but comparative studies are skewed by the fact that Reston virus infection can be lethal for NHPs. Here we used HLA-A2-transgenic, NOD-scid-IL-2 $\gamma$ receptorknockout (NSG-A2) mice reconstituted with human hematopoiesis to compare Ebola virus and Reston virus pathogenesis in a human-like environment. While markedly less pathogenic than Ebola virus, Reston virus killed $20 \%$ of infected mice, a finding that was linked to exacerbated inflammation and viral replication in the liver. In addition, the case fatality ratios of different Ebolavirus species in humans were recapitulated in the humanized mice. Our findings point to humanized mice as a putative model to test the pathogenicity of newly discovered filoviruses, and suggest that further investigations on Reston virus pathogenesis in humans are warranted.

Conflict of interest: The authors have declared that no conflict of interest exists.

Submitted: November 7, 2018 Accepted: September 19, 2019 Published: September 24, 2019

Reference information: /CI Insight. 2019;4(21):e126070. https://doi.org/10.1172/jci. insight.126070.

Copyright: (c) 2019, Escudero-Pérez et al. This is an open access article published under the terms of the Creative Commons Attribution 4.0 International License.

\section{Introduction}

Filoviruses of the genus Ebolavirus comprise 6 known species. The most virulent for humans is Ebola virus (species Zaire ebolavirus [EBOV]), which has caused most of the outbreaks to date, including the West African epidemic of 2013-2016 (1) and the ongoing epidemic in the Democratic Republic of the Congo (DRC) (2). Two other members of the genus, Sudan virus (Sudan ebolavirus [SUDV]) and Bundibugyo virus (Bundibugyo ebolavirus [BDBV]), are also pathogenic for humans, with reported case fatality rates (CFRs) of $50 \%$ and $25 \%$, respectively $(3,4)$. There is significantly less knowledge regarding the putative pathogenicity of Taï Forest virus (Taï Forest ebolavirus [TAFV]) and Reston virus (Reston ebolavirus [RESTV]) in humans. There is only one reported case of the former, a survivor $(5,6)$, and reports of seroconversion in the absence of disease for the latter $(7,8)$. The recent discovery of additional filoviruses and filovirus sequences in bats and other species (9-11) has underscored the need for animal models to test the putative pathogenicity of emerging filoviruses.

Nonhuman primates (NHPs), in particular rhesus and cynomolgus macaques, are the gold-standard models for the study of filovirus pathogenesis. Infection of NHPs with EBOV and SUDV reproduces many of the features of Ebola virus disease (EVD) in humans, and therefore, NHPs are preferred models for the development of vaccines and therapeutics $(12,13)$. However, this model presents limitations for comparative filovirus pathogenesis studies, since NHPs are also highly susceptible to RESTV and TAFV $(14,15)$.

We have previously shown that severely immune-compromised mice harboring human hematopoiesis are highly susceptible to EBOV infection (16). This model is based on the reconstitution of HLA-A2-transgenic NOD-scid-IL-2 $\gamma$ receptor-knockout mice with $\mathrm{CD}_{3} 4^{+}$human hematopoietic stem cells (HSCs) isolated from cord blood of HLA-matched donors (hereafter referred to as huNSG-A2 mice). Upon infection with WT EBOV (Mayinga variant), huNSG-A2 mice recapitulated many features of human disease including the incubation period, high lethality, the viremia, and high levels of serum aminotransferases (16). 
Here we show that upon mucosal infection, EBOV was significantly more pathogenic than RESTV in huNSG-A2 mice. However, 20\% of infected mice also died from RESTV infection. A comparative assessment of EBOV and RESTV pathogenesis indicated that lethal RESTV infection in this model was associated to exacerbated inflammation and sustained virus replication in the liver. These results suggest that under specific host conditions (e.g., immune suppression), RESTV may cause disease in humans. Moreover, the susceptibility of huNSG-A2 mice to viruses representative of different Ebolavirus species mimics that observed in humans, suggesting that mice harboring human immune components could serve as models to test the putative pathogenicity of newly discovered filoviruses.

\section{Results}

Mucosal RESTV replication kinetics is delayed with respect to that of EBOV. The natural portals of entry of ebolaviruses in humans are the skin and the mucosae (17). Therefore, we first evaluated the presence of human mature immune cells in the skin and mucosae of huNSG-A2 mice 12 weeks after transplantation of human CD34+ HSCs. Flow cytometry-based immunophenotyping showed that, indeed, mature antigen-presenting cells including human DCs and monocytes were observed in mouse lung and skin in the steady state (Figure 1A). In particular, the lung showed consistent reconstitution of human myeloid and lymphoid cell subsets, and thus we decided to use the intranasal route to mimic exposure to viruses via the respiratory mucosa.

We next performed an analysis of the infection kinetics of EBOV and RESTV in the respiratory mucosa in vivo. Histopathological analysis of lung samples using antibodies against human CD45 (hCD45), a pan-leukocyte marker, and the Ebolavirus nucleoprotein (NP), revealed stark differences in the replication kinetics of both viruses. On day 5 after infection, we already observed staining of EBOV NP in macrophage-like cells within the lung parenchyma, which colocalized with hCD45 (Figure 1B). On day 8 after infection, discrete clusters of EBOV replication were observed in the lung parenchyma. Conversely, replication of RESTV was significantly delayed and was not detectable prior to day 8 after infection (Figure 1B). These differences were not dependent on the levels of hCD45 cells, which were comparable in RESTV- and EBOV-infected mice (Figure 1C).

These results are in agreement with RESTV having slower replication kinetics in cell culture than EBOV (18). The colocalization pattern observed also suggests that both viruses have a preference for human as opposed to mouse cells. Indeed, both viruses replicated to substantially higher titers in human macrophages and DCs compared with mouse macrophages and DCs (Supplemental Figure 1; supplemental material available online with this article; https://doi.org/10.1172/jci.insight.126070DS1). As expected, in these in vitro assays, RESTV replication was also slower than that of EBOV.

Taken together, our data suggested that mucosal exposure to EBOV and RESTV in mice reconstituted with human HSCs could serve as a model to study filovirus pathogenesis in a human-like environment and to dissect the mechanisms responsible for differences in filovirus pathogenicity.

Infection of huNSG-A2 mice with ebolaviruses mimics species-specific CFRs. To determine whether infection of huNSG-A2 mice via mucosal exposure also recapitulated the lethality of other Ebolaviruses in humans, we sought to compare their susceptibility to 3 additional Ebolavirus species: SUDV, BDBV, and TAFV. Infection of huNSG-A2 mice with EBOV resulted in $92.86 \%$ lethality, comparable to that in our previous study using the intraperitoneal route (16). Similarly, infection with SUDV resulted in $71.43 \%$ lethality, which recapitulated the reported CFR of this virus in humans $(3,4)$ (Figure 2A). The high lethality of EBOV and SUDV was also associated with high morbidity (weight loss) in the model (Figure 2B). Infection with BDBV caused death in $28.58 \%$ of infected mice, which was also in agreement with the reported CFR in the only human outbreak described to date (19). TAFV infection killed $18.18 \%$ of infected mice, indicating that, while less pathogenic, TAFV may cause severe disease in our model. While significantly less pathogenic, RESTV infection resulted in death of $20 \%$ of infected mice, an outcome that was comparable to that of TAFV infection (Figure 2, A and B).

Among the main predictors of outcome of ebolavirus infection in humans are levels of viremia and serum aminotransferases (20-22). Thus, we compared the kinetics of viremia and AST in surviving and lethally infected huNSG-A2 mice. As expected, all surviving mice controlled viremia and maintained low levels of serum AST independently of the virus species to which they were exposed (Figure 2, C and D). Conversely, mice that died from infection had high levels of AST and virus in blood until death (Figure 2, C and D). An important difference was observed in the case of mice infected with TAFV. Mice that died from TAFV infection showed high levels of circulating AST (Fig- 
A Lung
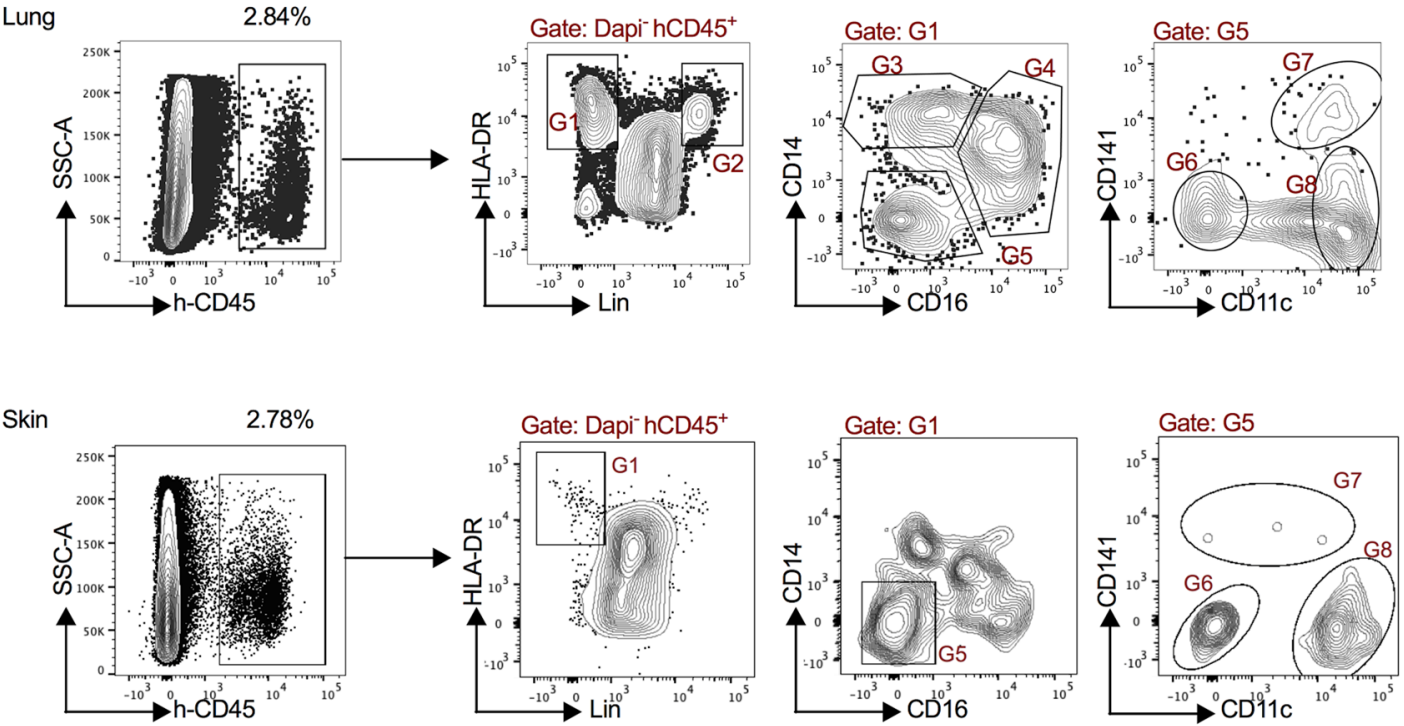

B
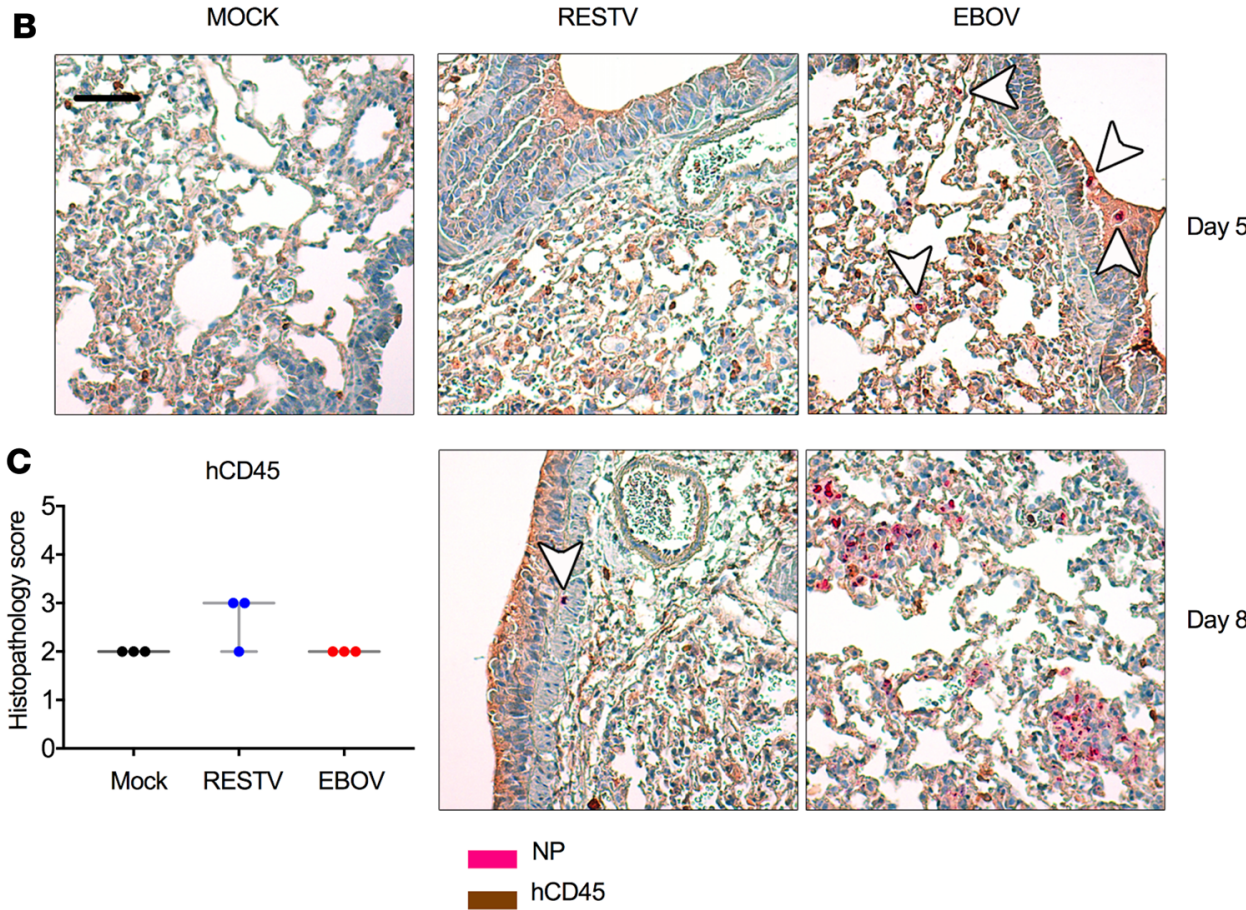

Day 8

Figure 1. Mucosal exposure of huNSG-A2 mice to EBOV and RESTV. (A) Flow cytometry-based evaluation of the presence of mature human immune cells in skin (lower back area) and lung of huNSG-A2 mice. Gates indicate the percentage of cells expressing human CD45 (h-CD45) in either organ. The gating strategy in the right panels shows the presence of human antigen-presenting cells (APCs) (G1), B cells (G2), CD14+ monocytes (C3), CD16 $6^{+}$monocytes (C4), nonmonocytic APCs (C5), and human DC subsets (G6-G8). (B) Histopathological analysis of huNSG-A2 lung tissue after infection with EBOV or RESTV on the indicated days after infection. White arrowheads indicate the presence of infected cells, showing EBOV NP- and CD45-positive staining. Scale bar: $50 \mu \mathrm{m}$ (C) Histopathology score (ordinal method, values of 0 to 5) assessing the levels of hCD45 staining in $n=3$ lung sections of RESTV- and EBOV-infected and control (Mock) mice. Boxand-whisker plots represent minimum to maximum values. All scoring values are shown.

ure 2D); however, the overall level of TAFV in blood was significantly lower than that observed for the other ebolaviruses (Figure 2C). Apart from its reported high lethality in chimpanzees $(15,23)$, little is known about TAFV pathogenesis in humans and NHPs. Our model suggests that at least the kinetics of replication of this virus differs from that of other species in the genus.

Previous reports have indicated differences in pathogenesis between the Mayinga and Makona variants of EBOV, in humans $(21,24)$ as well as NHPs and immunodeficient mice $(25,26)$. Thus, we next 
A

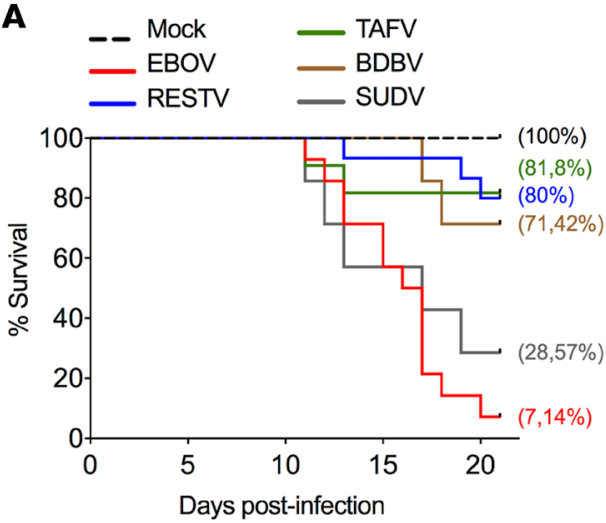

C
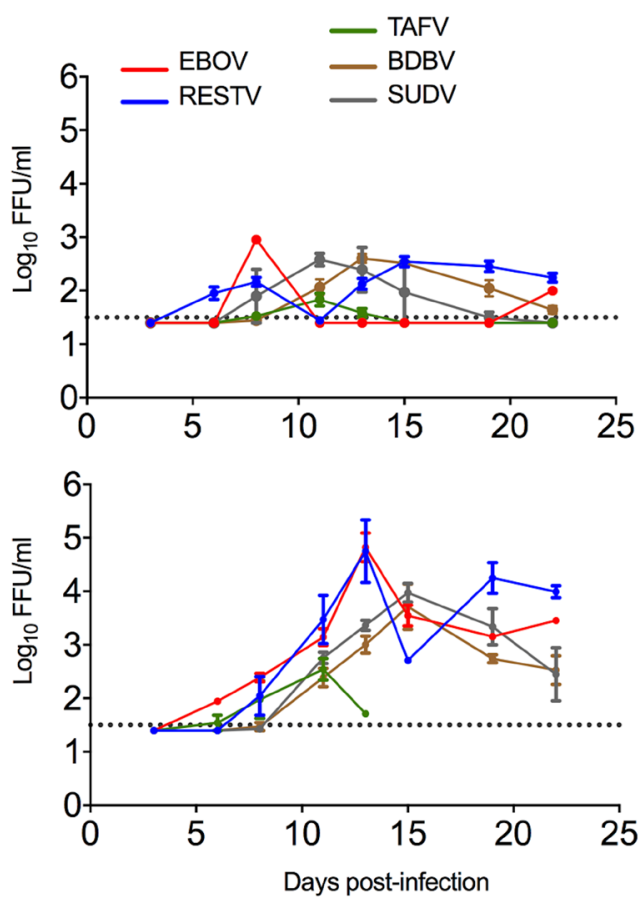

$\mathbf{E}$

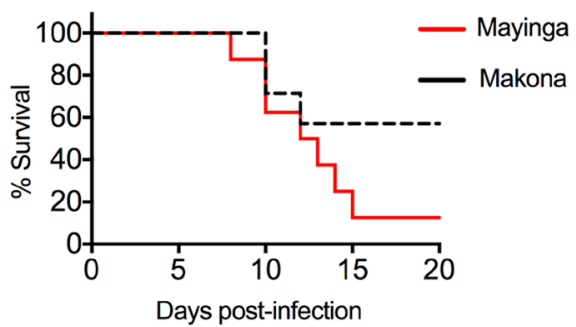

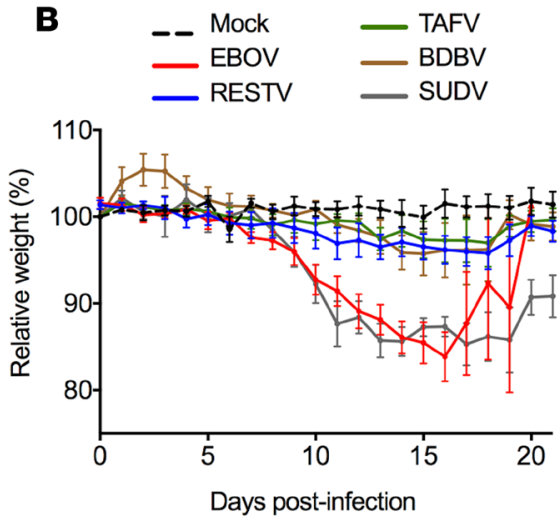

D
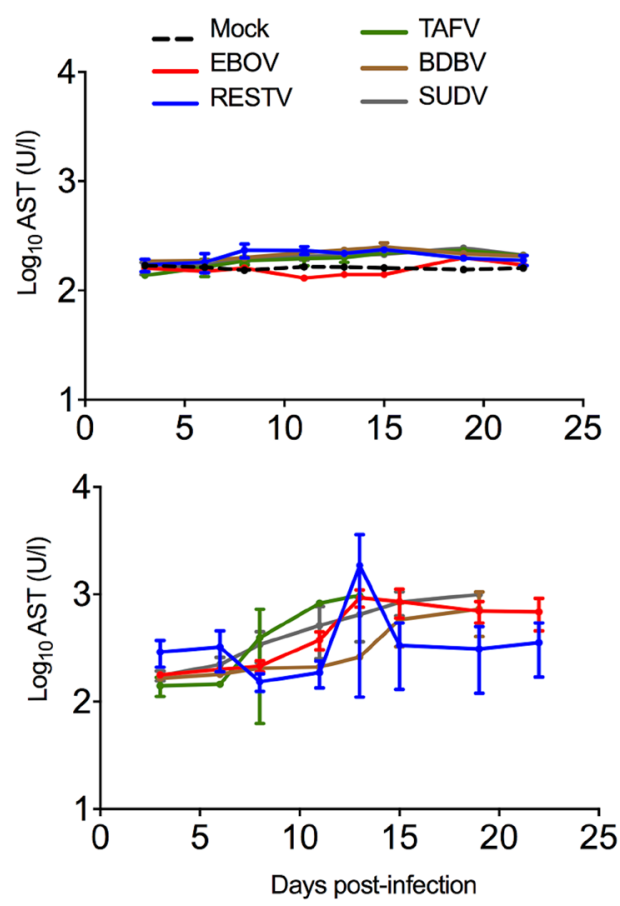

$\mathbf{F}$

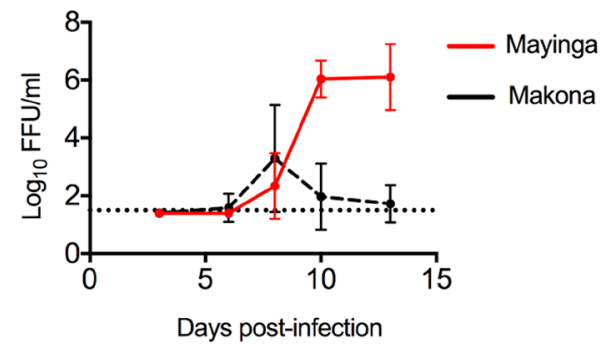

Figure 2. Comparative ebolavirus pathogenesis in huNSG-A2 mice. (A) Kaplan-Meier survival curves of infected mice. Mice were infected intranasally with 1000 FFU EBOV ( $n=14)$, RESTV ( $n=15)$, TAFV $(n=11)$, BDBV $(n=7)$, and SUDV $(n=7)$. Mock-infected mice $(n=11)$ received $20 \mu$ L PBS. Log-rank (Mantel-Cox) analysis indicated statistically significant differences between results for EBOV- and SUDV-infected mice and those for all the other groups $(P<0.0001)$. (B) Weight loss of infected huNSG-A2 mice. Nonparametric Kruskal-Wallis analysis followed by Dunn's post hoc test analysis indicated significant differences between results for mice infected with EBOV $(P=0.025)$ and SUDV $(P=0.017)$ and those for the other groups. (C) Kinetics of viremia in surviving and nonsurviving mice from infection to experimental endpoint. Dotted lines represent the limit of detection of $50 \mathrm{FFU} / \mathrm{mL}$. (D) Levels of AST in blood of infected mice (survivors and nonsurvivors). In the survivor group,the mock treatment group is represented by the black dashed line. (E) Survival curve of huNSG-A2 mice infected with the EBOV variants Mayinga $(n=8)$ and Makona $(n=7)$. Log-rank (Mantel-Cox) analysis indicated statistical significance $(P=0.042)$. (F) Kinetics of viremia from infection to experimental endpoint. Dotted lines represent the limit of detection of $50 \mathrm{FFU} / \mathrm{mL}$. Throughout the figure, error bars represent mean $\pm \mathrm{SEM}$. 
determined whether our model would also recapitulate differences in pathogenesis between these 2 EBOV variants. Indeed, while EBOV Mayinga was highly lethal in huNSG-A2 mice and caused high levels of viremia, mice infected with EBOV Makona showed reduced lethality (50\%), as well as reduced levels of virus in blood (Figure 2, E and F).

In summary, our data indicated that upon mucosal exposure, huNSG-A2 mice showed distinct susceptibility to different Ebolavirus species and EBOV variants, suggesting that this model could provide insight into virus species-specific pathogenicity mechanisms and the putative virulence of newly discovered filoviruses.

The lethality of RESTV in humanized mice is associated with high inflammation. In order to investigate why RESTV infection was lethal for a subset of mice, we first performed Luminex-based analysis of cytokine production in RESTV- and EBOV-infected mice over the course of infection. To determine the specific contribution of the human immune system in our model, we utilized panels to assess the expression of human cytokines, chemokines, and coagulation markers.

Expression of high levels of proinflammatory cytokines at early time points after infection predicted fatal outcome in mice infected with either EBOV (Mayinga) or RESTV. Thus, on day 3 after infection, we observed high levels of proinflammatory chemokines (e.g., macrophage inflammatory proteins-1 $\alpha$ [MIP-1 $\alpha$, MIP- $1 \beta$, IL-8, IP-10), IFNs, markers of endothelial dysfunction (E-selectin, Pecam-1), and coagulation markers (e.g., D-dimers) in mice that died from either EBOV or RESTV infection (Figure 3A). Levels of proinflammatory cytokines in serum were, however, significantly higher in mice that died from RESTV infection and were maintained until day 10 after inoculation, indicating sustained inflammation over the course of infection (Supplemental Figure 2). Overall, the highest levels of proinflammatory mediators were observed in mice with high levels of viremia, elevated levels of serum AST, and short time to death after exposure (Table 1). These data strongly suggest that the severity of EBOV infection in huNSG-A2 mice can be monitored using the same biomarkers proposed for humans, namely, levels of viremia and inflammation. Our results also indicated that death from either EBOV or RESTV infection was associated with high levels of expression of proinflammatory cytokines and elevated levels of serum aminotransferases, suggesting liver damage.

The lethality of RESTV in humanized mice is associated with high levels of virus replication in the liver. To further assess the relationship among human immune cell function, virus replication, and liver damage, we performed histopathological analysis of tissues during necropsies of RESTV-infected and EBOV-infected mice. Interestingly, while histopathology revealed stark differences between mice that survived RESTV infection and those that died, these differences were restricted to the liver. Mice infected with RESTV showed similar levels of virus replication in spleen and lung, which was independent of the outcome. Mice that survived RESTV showed even higher levels of virus replication in the kidney than their lethally infected counterparts. However, mice that died from EBOV or RESTV infection showed significantly higher levels of virus replication in the liver, whereas RESTV-surviving mice controlled virus replication in this organ (Figure 4A). These high levels of virus replication in lethally infected mice (EBOV and RESTV) correlated with high levels of infiltration in the liver of immune cells including human B lymphocytes and macrophages/monocytes (Figure 4B). Of note, in RESTV survivors, we observed active phagocytosis of infected RESTV NP ${ }^{+}$cells by Iba1 ${ }^{+}$monocytes/macrophages (Figure 4C, inset). Although indirectly, these findings strongly suggest that virus replication in these mice may be associated with infiltration of myeloid cells that support virus replication. Lethality caused by either EBOV or RESTV was also associated with higher levels of caspase-3 staining in the tissue sections (Figure 4C). These results indicated that fatal EBOV and RESTV infection in huNSG-A2 mice was characterized by higher levels of virus replication in the liver, infiltration of immune cells, and increased levels of apoptosis.

\section{Discussion}

In this study, we present a mouse model with mature human peripheral immune cells that is susceptible to infection with nonadapted ebolaviruses. We utilized intranasal inoculation of virus in order to mimic mucosal exposure, a probable route of infection during human EVD outbreaks $(27,28)$. Recently, pathogenesis studies using the mucosal route of exposure to EBOV have also been conducted in NHPs and guinea pigs. In both models, intranasal inoculation of EBOV resulted in delayed time to death compared with intramuscular inoculation $(29,30)$. In another NHP study, oral and conjunctival exposure to low doses of EBOV resulted in survival (31). These findings strongly suggest that mucosal immunity may play an important role in precluding systemic dissemination of EBOV from the initial points of infection. However, in the humanized mouse model utilized in our study, we did not observe differences in lethality or time 


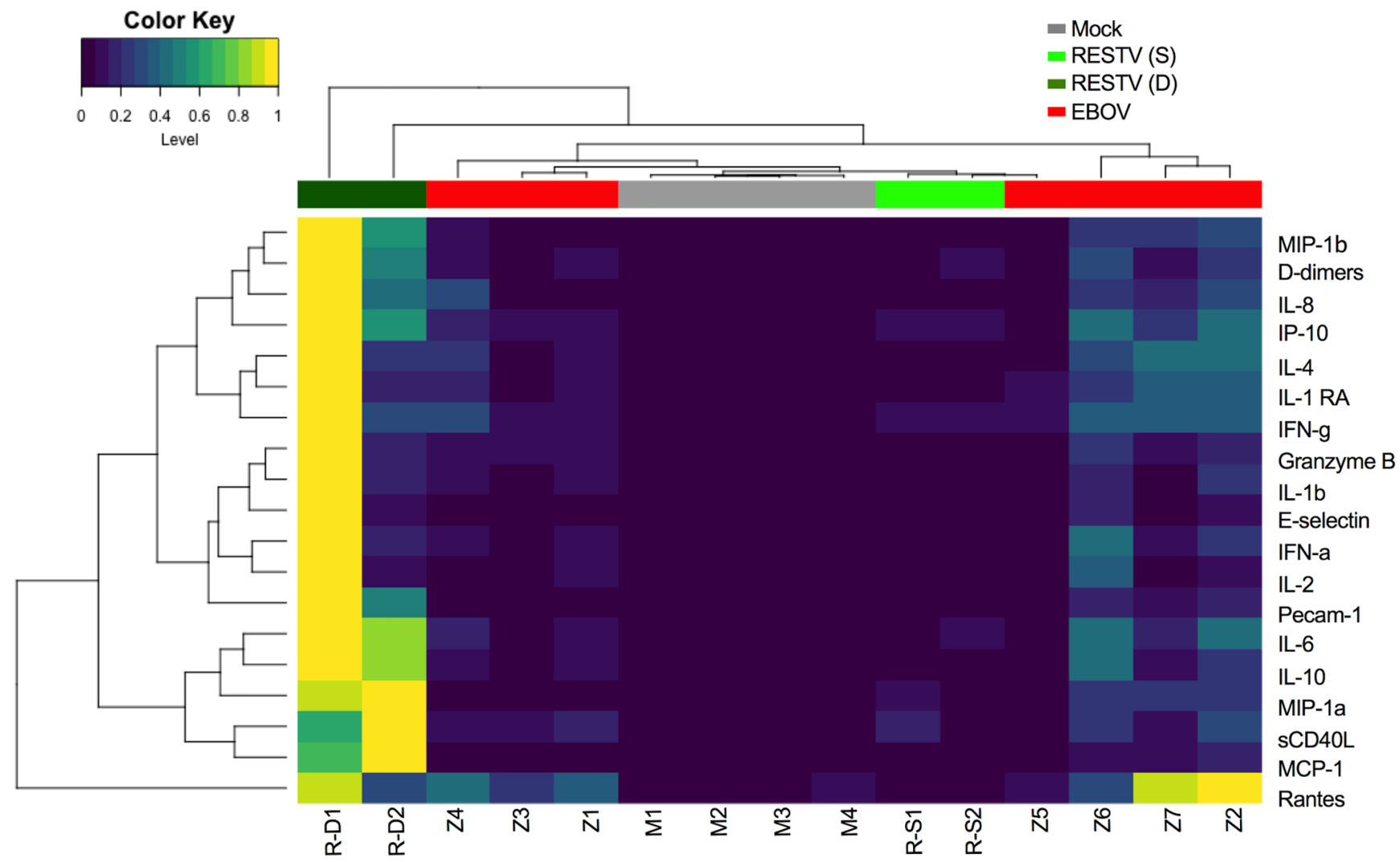

Figure 3. Inflammatory profile of mice infected with EBOV (Mayinga variant) and RESTV. Heatmap showing levels of the indicated analytes in plasma of mice infected with either EBOV $(Z, n=7)$ or RESTV (R-D, dead; R-S, survivor; $n=4)$ on day 3 after infection. Hierarchical clustering of samples was performed based on euclidean distance using complete linkage. Cytokine data collected via Luminex multiplexed ELISA assays were normalized using min-max normalization, which normalized cytokine values between 1 and 0 . Mock-infected mice $(M)$ that received PBS are shown as controls. Visualization of cytokine profiles was performed using the $\mathrm{R}$ function heatmap. 2 implementing euclidean distance and using ward.D clustering.

to death after infection when comparing intranasal and intraperitoneal inoculation of virus (16). These findings probably reflect limitations in the immune responses in our model, such as defects in immune cell migration to tissue-draining lymph nodes and poor $\mathrm{CD} 4^{+} \mathrm{T}$ cell help.

NSG mice transplanted with human CD34 ${ }^{+}$HSC have been previously shown to mount human-like immune responses to viral infections, including those caused by HIV, Ebola virus, dengue virus, and adenovirus, among others (32-35). Myeloid and T cell activation as well as production of human immunoglobulins have been described in humanized NSG mice. In the HLA-A2-transgenic model, virus-specific $\mathrm{CD}^{+} \mathrm{T}$ cell responses have been also demonstrated $(34,35)$, although in the case of experimental dengue infection, they resulted in inefficient virus clearance (35). In our study, however, we observed that mice surviving infection were able to control virus loads and, in some cases (i.e., SUDV and BDBV) clear viremia. We hypothesize that such control is probably dependent to a great extent on HLA-A2-restricted CD8 ${ }^{+} \mathrm{T}$ cells similarly to what has been previously described for acute adenovirus infection in the same model (34). This would be also consistent with our previous observation that nontransplanted NSG-A2 mice experience a lengthy chronic disease characterized by sustained viremia (16). Although further experiments are needed to test this hypothesis, this would be also in line with the important role of $\mathrm{CD}^{+} \mathrm{T}$ cell-mediated immunity in recovery from acute EVD (24). However, as mentioned above, there are also several limitations of the NSG model, including poor $\mathrm{CD}^{+} \mathrm{T}$ cell-dependent responses, long-term problems associated to graft-versus-host disease, hemophagocytic lymphohistocytosis, and lack of immunoglobulin isotype switching $(36,37)$. Despite the latter, huNSG-A2 mice that died from EBOV or RESTV infection showed evidence of recruitment of human macrophages and B cells to the sites of infection, in particular to the liver. These results are consistent with the massive generation of activated $\mathrm{CD} 20^{+} \mathrm{B}$ cells after EBOV infection in humans (38) and their recruitment to infection sites (39). 
Table 1. Individual pathogenesis data of all mice included in the heatmap analysis in Figure 3

\begin{tabular}{lccc}
\hline & TOD (d) & Viremia $\left(\log _{10}\right.$ FFU/mL) & AST (log $\left.\mathbf{l}_{10} / \mathbf{L}\right)$ \\
EBOV (Z5) & 20 & 3.45 & 1120 \\
EBOV (Z3) & 17 & 3.01 & 1983 \\
EBOV (Z4) & 16 & 3.04 & 1140 \\
EBOV (Z1) & 18 & 3.24 & 1120 \\
EBOV(Z6) & 12 & 5.89 & 2340 \\
EBOV (Z2) & 11 & 5.42 & 1290 \\
EBOV (Z7) & 15 & 4.55 & 1340 \\
RESTV (R_D1) & 12 & 5.98 & 5370 \\
RESTV (R_D2) & 19 & 4.53 & 1540 \\
RESTV (R-S1) & N/A & $3.19^{\mathrm{A}}$ & $220^{\mathrm{A}}$ \\
RESTV (R_S2) & N/A & $2.74^{\mathrm{A}}$ & $240^{\mathrm{A}}$
\end{tabular}

TOD, time to death after infection; N/A, not applicable. ${ }^{A}$ Maximum values over the course of infection in surviving mice.

In our study we also observed that lethality associated with either EBOV or RESTV infection was associated with early and sustained production of proinflammatory cytokines, high levels of viremia, and high levels of AST. These findings are in agreement with data collected during human outbreaks that indicated cycle threshold $(\mathrm{Ct})$ values (as a surrogate of viremia) and inflammation as biomarkers with predictive potential $(20,22,40)$. Interestingly, mice that died from infection with either virus also showed early upregulation of D-dimers, which suggested that fatal ebolavirus infection in humanized mice is also associated with coagulopathy. This observation is in line with the presence of focal bleeding in necropsies of EBOV-infected huNSG-A2 mice (16).

The reasons for the stark differences in EBOV and RESTV pathogenicity are unknown. Several factors have been proposed, including the ability of the virus glycoprotein ability to direct entry into target cells (41) and perhaps the efficacy of viral protein 35 (VP35) and VP24 to counteract the type I IFN response in infected cells $(42,43)$. Presumably for these reasons, RESTV replication in cell culture is delayed with respect to that of EBOV (18). In this study using mice harboring human peripheral immune cells, we have shown that these differences in replication kinetics are also present in vivo, and that both EBOV and RESTV have a preference for human cells. Our findings are consistent with recently published data demonstrating that lethal EBOV infection and nonlethal RESTV infection are characterized by substantial differences in virus replication in the liver (44) and strongly support the notion that liver pathology is a central feature of lethal filovirus infection $(21,45,46)$. However, our data also indicate that under circumstances whereby RESTV is able to colonize the liver, this may lead to lethal infection. We hypothesize that the mucosal barrier may be of great importance for controlling early RESTV replication. However, this barrier can be overcome, leading to virus dissemination, high levels of inflammation, coagulopathy, and high levels of virus replication in the liver.

Finally, we propose that the model presented here may be of use to test the putative pathogenicity of newly discovered filoviruses. Even though further studies are needed to dissect the pathogenic features of ebolaviruses in huNSG-A2 mice, our initial studies indicate that the susceptibility of these mice to specific ebolaviruses was very similar to that of humans. Moreover, within the Zaire ebolavirus species, huNSG-A2 mice were significantly less susceptible to Makona virus compared with Mayinga virus, which was in agreement with reported CFRs in human $(17,21)$ and animal model studies $(25,26)$.

\section{Methods}

Generation of huNSG-A2 mice. Humanized mice were generated as previously described (16). Briefly, CD34+ human HSCs were positively selected (EasySep Human CD34 Positive Selection Kit, StemCell Technologies) from cord blood. To reconstitute the human hematopoietic system in NSG-A2 mice, we utilized the NOD.Cg-Prkdc sid Il2rg ${ }^{\text {tml } W_{j l}} \mathrm{Tg}$ (HLA-A2.1)1Enge/SzJ mouse strain purchased from the Jackson Laboratory. All experiments were conducted with 5-week-old NSG-A2 (HLA-A2.1) females conditioned by sublethal irradiation (240 cGy). Four hours after irradiation, mice underwent intravenous (retro-orbital) transplantation of $10^{6}$ HLA-A2-matching CD34 ${ }^{+}$HSCs per mouse. Eight weeks after HSC transplantation, blood samples were collected, and the presence of human hematopoietic cells was quantified by flow cytometry using anti-human CD45 antibody BV510 (clone HI30; 304035, BioLegend) and anti-mouse CD45.2 anti- 
A
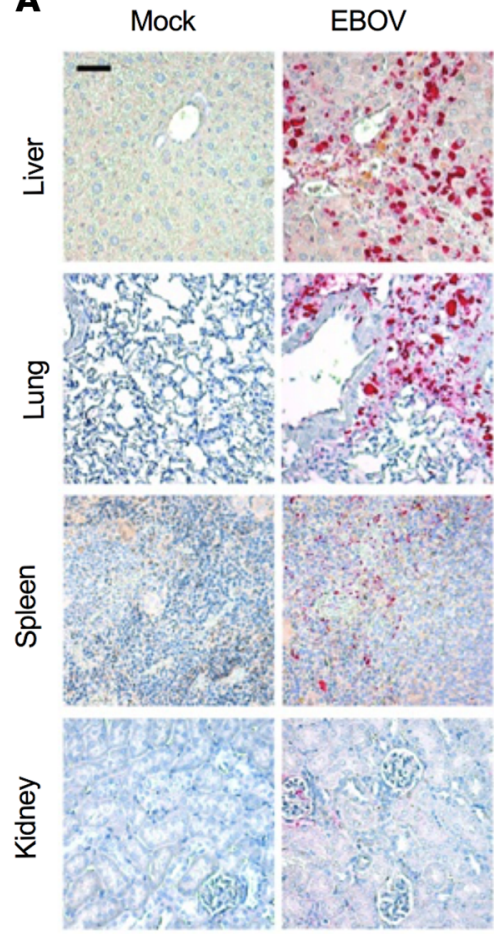

RESTV (S)
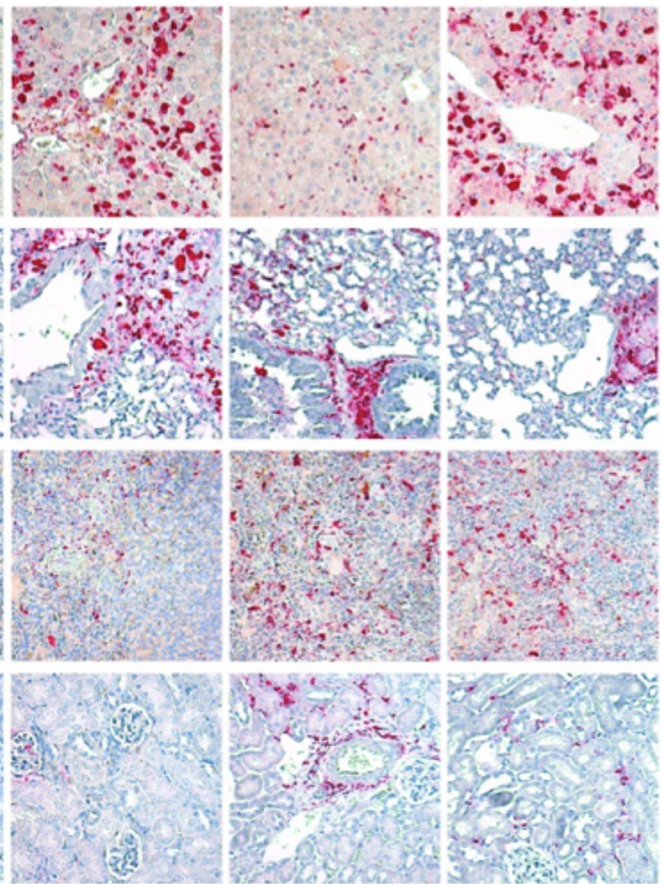

C

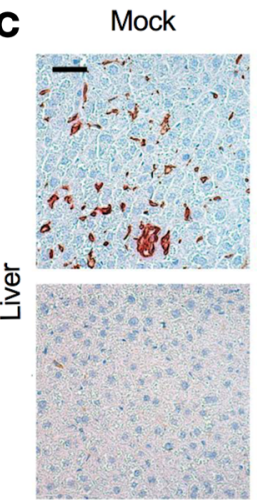

EBOV
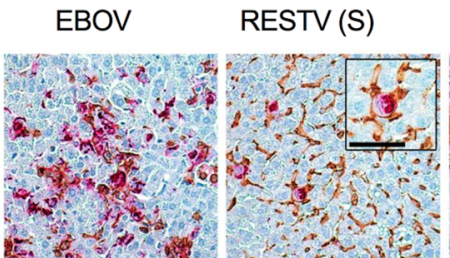

RESTV (D)
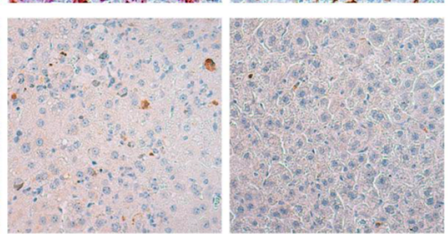

B

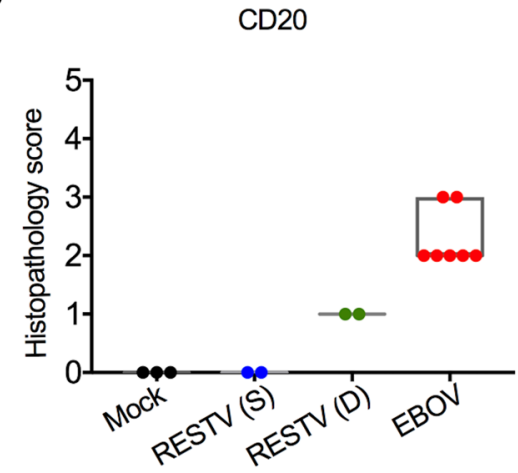

lba1

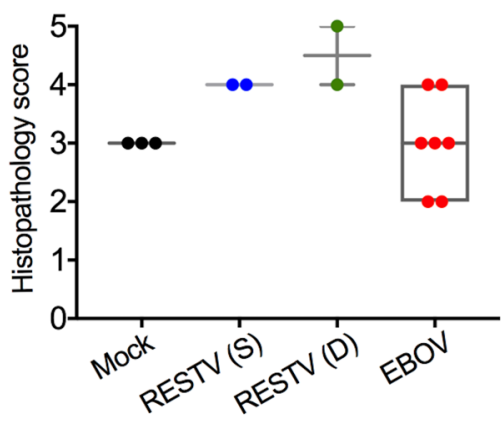

NP

Iba1

Figure 4. Liver pathology of EBOV- and RESTV-infected mice. (A) Histopathological findings in tissue sections of huNSG-A2 mice infected with EBOV and RESTV. In lethally infected mice, pathological assessment was done at the time of death (necropsy); surviving mice were euthanized on day 30 after infection. Red indicates staining of EBOV and RESTV NP in the indicated tissues. (B) Histopathological score (ordinal method, values of 0 to 5 ) for human CD20- and Iba1-positive cells in liver sections of mock-infected ( $n=3)$, RESTV-infected/surviving (S) ( $n=2)$, lethally RESTV-infected (D) ( $n=2)$, and EBOV-infected ( $n=7$ ) mice. Box-and-whisker plots represent minimum to maximum values. All scoring values are shown. (C) Histopathological analysis of liver sections subjected to immunohistochemistry staining with anti-NP (red), anti-lba1 (brown) and anti-caspase-3 (Casp 3; brown) antibodies. The magnified image inside the square shows an infected macrophage surrounded by lba1+ cells. Scale bars: $50 \mu \mathrm{m}$. Mock-infected mice received $20 \mu \mathrm{L}$ PBS.

body PerCP/Cy5.5 (clone 104; 109825, BioLegend). Samples were analyzed in a LSR II Fortessa flow cytometer (BD Biosciences). Mice containing 30\%-50\% HSPCs were selected for all the experiments. All infection experiments were performed at week 9-10 after engraftment.

Mouse infection and disease monitoring. Mice were intranasally infected with 1000 focus-forming units (FFU) of EBOV (Ebola virus/H.sapiens-tc/COD/1976/Yambuku-Mayinga and Ebola virus/H.sapiens-wt/GIN/2014/Makona-C07). RESTV (Pennsylvania strain) and TAFV (Tai Forest virus/H.sapiens-tc/CIV/1994/Pauleoula-CI) were donated by Stephan Becker (Philipps University of Marburg,Marburg, Germany). BDBV (Bundibugyo virus/H.sapiens-tc/UGA/2007/Bundibugyo-200706291) and SUDV (Sudan virus/H.sapiens-tc/UGA/2000/Gulu-808892) were donated by Christina Spiropoulou (Centers for Disease Control and Prevention, Atlanta, Georgia, USA). All virus stocks were tested for mycoplasma (MycoAlert Mycoplasma Detection Kit, Lonza) and certified mycoplasma free. 
A mock treatment group of mice received PBS and was kept as a negative control. All mice were kept in individually ventilated cages inside a biosafety level 4 (BSL4) laboratory at the Bernhard Nocht Institute and fed autoclaved food and water. Mice were monitored (weight, temperature, and general body scoring) daily over the course of the disease. According to the guidelines approved for our study, animals with severe signs of disease such as bleeding, lethargy, temperature lower than $28^{\circ} \mathrm{C}$, or weight loss of more than $20 \%$ of their original weight were euthanized.

Immunofocus assay. Infectious virus particles in blood and organ samples were determined by immunofocus assay. Organs were weighted and homogenized in tubes containing $1 \mathrm{~mL}$ DMEM using Lysing Matrix D tubes (MP Biomedicals) containing a bead mill. Vero 81 (ATCC CCL-81) cells seeded in 24-well plates were incubated with $200 \mu \mathrm{L}$ of serial 10-fold dilutions of homogenized organs and blood samples. The inoculum was removed after 1 hour and replaced with a 1\% methylcellulose medium overlay. After 6 days of incubation, overlay was removed, and cells were fixed with $4 \%$ formaldehyde, washed with water, and permeabilized with $0.5 \%$ Triton X-100 in PBS. After washing with PBS and blocking with 5\% FCS in PBS, cell foci were detected using an in-house-generated mouse polyclonal anti-pan-Ebolavirus NP primary antibody $\left(1: 2000\right.$ overnight at $\left.4^{\circ} \mathrm{C}\right)$. For detection of foci, cells were incubated with secondary peroxidase-conjugated AffiniPure Sheep Anti-Mouse IgG (H+L) antibody (515-035-003, Jackson ImmunoResearch Laboratories Inc.) at a 1:40,000 dilution for 45 minutes at room temperature. After washing with water, foci were visualized with tetramethylbenzidine (TMB) and counted.

Replication assay. PBMCs were isolated from the blood of healthy donors by centrifugation in a lymphocyte separation medium (Ficoll). The recovered monocyte fraction was depleted of cell contaminants by $\mathrm{CD} 14^{+}$selection (Miltenyi Biotec), ensuring purification rates of $\geq 95 \%$. Immature DCs and monocyte-derived macrophages were obtained upon incubation of cells in complete RPMI-1640 (Life Technologies) supplemented with $200 \mathrm{mM}$ L-glutamine (Life Technologies), $10 \mathrm{mM}$ HEPES buffer (Life Technologies), 10\% decomplemented FCS, and $8 \mu \mathrm{g} / \mathrm{mL}$ Gentamicin (Life Technologies) for 5 days with $50 \mathrm{ng} / \mathrm{mL}$ GM-CSF (PeproTech) and $50 \mathrm{ng} / \mathrm{mL}$ IL-4 (PeproTech) or $40 \mathrm{ng} / \mathrm{mL}$ M-CSF (PeproTech), respectively. Monocyte-derived human DCs and macrophages were then infected at an MOI of 0.5 as described above.

For generation of murine DCs and macrophages, murine bone marrow was extracted from 8-week-old male WT C57BL/6 mice (The Jackson Laboratory). Briefly, mice were euthanized, and bone marrow from tibiae and femurs was harvested by cutting the edges of the bones and flushing with a $29 \mathrm{G}$ needle in sterile conditions. Then, red blood cells were lysed (BD Pharm Lysing Buffer), and cells were seeded at a density of $10^{6}$ cells $/ \mathrm{mL}$ in 24 -well plates. For differentiation into murine DCs, cell culture medium (RPMI with $10 \%$ FCS) was supplemented with $50 \mathrm{ng} / \mathrm{mL}$ GM-CSF (PeproTech). For differentiation of bone marrow progenitors into macrophages, the medium was supplemented with $20 \mathrm{ng} / \mathrm{mL} \mathrm{M-CSF}$ (Miltenyi Biotec). Each cell type was then infected in triplicate at an MOI of 0.01 with EBOV or RESTV or with $2.5 \%$ FCS supplemented RPMI as mock control. Infectious viral particles were quantified daily in tissue culture supernatants using immunofocus assays as described above.

Clinical chemistry. Serum samples from infected mice were diluted 1:10 in water, and quantification of serum aminotransferases (AST) was determined by using commercially available GOT/AST Fuji DRICHEM slides in Fujifilm in a DRI-CHEM NX500 analyzer. The limit of detection for AST was $10 \mathrm{U} / \mathrm{L}$.

Flow cytometry. Single-cell suspensions were prepared from organs by cutting tissues into small fragments, followed by enzymatic digestion for 30 minutes at $37^{\circ} \mathrm{C}$ with Collagenase $\mathrm{D}(2 \mathrm{mg} / \mathrm{mL})$ (Roche) and DNAase $(50 \mu \mathrm{g} / \mathrm{mL})$ in RPMI-1640 medium. Tissue fragments were further disrupted by passage through a $70-\mu \mathrm{m}$ nylon cell strainer (BD Biosciences). Red blood cells were lysed with BD Pharm Lyse buffer (BD Biosciences). Cells were then stained for viability with Zombie NIR (BioLegend) for 30 minutes at room temperature, washed twice with PBS, and blocked with Human TruStain FcX (Fc receptor blocking solution, BioLegend) for 20 minutes at room temperature. Cells were stained with an antibody cocktail consisting on the following multiparametric flow cytometry panel: CD45-FITC (2D1; 368507, BioLegend), CD1c-APC (L161; 331523, BioLegend), CD38-PerCP-Cy5.5 (HIT2; 303521, BioLegend), CD16-PECy7 (3G8; 302015, BioLegend), CD141-PE (M80; 344103, BioLegend), HLADR-BV785 (L243; 307641, BioLegend), CD8-BV570 (RPA-T8; 301037, BioLegend), CD14-BV510 (M5E2; 301841, BioLegend), CD56 (HCD56; 318325, BioLegend), CD19-PB (HIB19; 982404 BioLegend), CD4-BUV737 (SK3; 564305, BD Biosciences), and CD3-BV650 (OKT3; 317323, BioLegend). Formaldehyde-inactivated samples were acquired using an LSRFortessa (BD Biosciences) and analyzed with FlowJo software. 
Histology and immunohistochemistry. Mouse tissue samples were fixed in 4\% formalin/PBS and were embedded in paraffin. Sections were then stained with H\&E or processed for immunohistochemistry as follows: After dewaxing and inactivation of endogenous peroxidases (PBS/3\% hydrogen peroxide), antibody-specific antigen retrieval was performed. Sections were blocked (PBS/10\% FCS) and incubated with primary antibodies for rat anti-mouse CD3 (T cells; OKT; 317301, BioLegend), anti-CD20 (B cells; Dako Omnis clone L26; GA604), anti-cleaved caspase-3 (apoptosis marker; ab2302, Abcam), anti-CD14 (monocytes; ab183322, Abcam), or anti-Ebola-NP (clone KZ51 IgG1 mouse; Absolute Antibody). Bound primary antibodies were detected with anti-mouse, anti-rabbit or anti-rat $N$-Histofine Simple Stain MAX PO immune-enzyme polymer (Nichirei Biosciences Inc.) and stained with DAB substrate using the ultraView Universal DAB Detection Kit (Ventana). Tissues were counterstained with hematoxylin.

Luminex multiplex assay. Sera from all mice were collected on days 3, 6, 9, 12, 15, 18, 21, and 23 after infection, and analysis of cytokine concentrations was performed using ProcartaPlex Multiplex Immunoassay Magnetic Bead Panel Premix 19 Plex (PPX-19) according to the manufacturer's instructions. A Luminex 200 system (Millipore) was used for data acquisition.

Heatmaps. Visualization of cytokine profiles was performed using the $\mathrm{R}$ function heatmap.2, implementing euclidian distance and using the ward.D clustering method to generate the dendrograms. Minmax normalization was performed for each cytokine to normalize data ranges between 1 and 0 .

Statistics. Statistical analyses were done using Graphpad Prism 6 software. All data are presented as mean \pm SEM. For multiple comparisons, nonparametric 1-way ANOVA on ranks (Kruskal-Wallis test) followed by Dunn's post hoc test was used. For comparison of survival curves, log-rank (Mantel-Cox) tests were used. A $P$ value less than 0.05 was considered significant.

Study approval. Human HSCs were purified from cord blood obtained at the Asklepios Klinic Nord in Hamburg. All patients agreed to donation of biological material by informed written consent under a protocol approved by the Ethics Commision of the Medical Association of Hamburg (WF-054/15). Animal experiments were performed under protocols approved by the German animal protection authorities (Behörde für Gesundheit und Verbraucherschutz, Hamburg, approval 110/17). All infection experiments were carried out in the BSL4 laboratory of the Bernhard Nocht Institute by experienced and highly trained personnel using positive pressure biocontainment suits.

\section{Author contributions}

BEP and CMF designed the experiments. BEP, PR, MR, AL, EVN, and JRP performed experiments. KH and SK performed histopathology experiments and analyzed the data. SGM, JMG, and ER generated humanized mice for the study. ER and CMF coordinated the study. BEP and CMF wrote the manuscript.

\section{Acknowledgments}

This work was supported by the Marie Skłodowska-Curie Actions (grant 708181, EBOPATH) of the European Commission and by the DZIF (grant TTU 01.702_00). This work also received support from the EU/European Federation of Pharmaceutical Industries and Associations (EFPIA)/Innovative Medicines Initiative 2 Joint Undertaking (grant 116088, PEVIA).

Address correspondence to: César Muñoz-Fontela, Bernhard Nocht Institute for Tropical Medicine, Bernhard Nocht Strasse 74, 20359 Hamburg, Germany. Phone: 49.4042818.541; Email: munoz-fontela@bnitm. de. Or to: Estefanía Rodríguez, Heinrich Pette Institute for Experimental Virology, Martinistrasse 52, 20251 Hamburg, Germany. Phone: 49.4048051.304; Email: estefania.rodriguez-burgos@leibniz-hpi.de.

PR's present address is: Department of Infectious Diseases, Leiden University Medical Center, Leiden, Netherlands.

1. Baize S, et al. Emergence of Zaire ebola virus disease in guinea. N Engl J Med. 2014;371(15):1418-1425

2. Munster VJ, et al. Outbreaks in a rapidly changing Central Africa - lessons from Ebola. N Engl J Med. 2018;379(13):1198-1201.

3. Centers for Disease Control Prevention (CDC). Outbreak of Ebola hemorrhagic fever Uganda, August 2000-January 2001. MMWR Morb Mortal Wkly Rep. 2001;50(5):73-77.

4. Team ROAWIS. Ebola haemorrhagic fever in Sudan, 1976. Report of a WHO/International Study Team. Bull World Health Organ. 1978;56(2):247-270.

5. Le Guenno B, et al. Isolation and partial characterisation of a new strain of Ebola virus. Lancet. 1995;345(8960):1271-1274. 
6. Formenty P, Hatz C, Le Guenno B, Stoll A, Rogenmoser P, Widmer A. Human infection due to Ebola virus, subtype Côte d'Ivoire: clinical and biologic presentation. J Infect Dis. 1999;179(suppl 1):S48-S53. 1999.

7. Centers for Disease Control and Prevention. Epidemiologic notes and reports updates: filovirus infection in animal handlers. MMWR Weekly. 1990;39(13);221. https://www.cdc.gov/mmwr/preview/mmwrhtml/00001593.htm. Reviewed May 2, 20019 Accessed September 30, 2019.

8. Miranda MEG, Miranda NLJ. Reston ebolavirus in humans and animals in the Philippines: a review. J Infect Dis. 2011;204(suppl 3):S757-S760.

9. Negredo A, et al. Discovery of an ebolavirus-like filovirus in Europe. PLoS Pathog. 2011;7(10):e1002304.

10. Goldstein T, et al. The discovery of Bombali virus adds further support for bats as hosts of ebolaviruses. Nat Microbiol. 2018;3(10):1084-1089.

11. Shi M, et al. The evolutionary history of vertebrate RNA viruses. Nature. 2018;556(7700):197-202.

12. Cross RW, Mire CE, Feldmann H, Geisbert TW. Post-exposure treatments for Ebola and Marburg virus infections. Nat Rev Drug Discov. 2018;17(6):413-434.

13. Geisbert TW, et al. Pathogenesis of Ebola hemorrhagic fever in cynomolgus macaques: evidence that dendritic cells are early and sustained targets of infection. Am J Pathol. 2003;163(6):2347-2370.

14. Jahrling PB, Geisbert TW, Jaax NK, Hanes MA, Ksiazek TG, Peters CJ. Experimental infection of cynomolgus macaques with Ebola-Reston filoviruses from the 1989-1990 U.S. epizootic. Arch Virol Suppl. 1996;11:115-134.

15. Formenty P, et al. Ebola virus outbreak among wild chimpanzees living in a rain forest of Côte d'Ivoire. J Infect Dis. 1999;179(suppl 1):S120-S126.

16. Lüdtke A, et al. Ebola virus disease in mice transplanted with human hematopoietic stem cells. J Virol. 2015; 89(8):4700-4704

17. Muñoz-Fontela C, McElroy AK. Ebola virus disease in humans: pathophysiology and immunity. Curr Top Microbiol Immunol. 2017;411:141-169.

18. Boehmann Y, Enterlein S, Randolf A, Mühlberger E. A reconstituted replication and transcription system for Ebola virus Reston and comparison with Ebola virus Zaire. Virology. 2005;332(1):406-417.

19. Towner JS, et al. Newly discovered ebola virus associated with hemorrhagic fever outbreak in Uganda. PLoS Pathog. 2008;4(11):e1000212.

20. Lanini S, et al. Blood kinetics of Ebola virus in survivors and nonsurvivors. J Clin Invest. 2015;125(12):4692-4698.

21. Schieffelin JS, et al. Clinical illness and outcomes in patients with Ebola in Sierra Leone. NEngl J Med. 2014; 371(22): 2092-2100.

22. La Vega de MA, et al. Ebola viral load at diagnosis associates with patient outcome and outbreak evolution. J Clin Invest. 2015;125(12):4421-4428.

23. Wyers M, et al. Histopathological and immunohistochemical studies of lesions associated with Ebola virus in a naturally infected chimpanzee. J Infect Dis. 1999;179(supp1 1):S54-S59.

24. Ruibal P, et al. Unique human immune signature of Ebola virus disease in Guinea. Nature. 2016;533(7601):100-104.

25. Marzi A, Feldmann F, Hanley PW, Scott DP, Günther S, Feldmann H. Delayed disease progression in cynomolgus macaques infected with Ebola virus Makona strain. Emerg Infect Dis. 2015;21(10):1777-1783.

26. Smither SJ, et al. Ebola virus Makona shows reduced lethality in an immune-deficient mouse model. J Infect Dis. 2016; 214(suppl 3):S268-S274.

27. Bausch DG, et al. Assessment of the risk of Ebola virus transmission from bodily fluids and fomites. J Infect Dis. 2007;196(suppl 2):S142-S147.

28. Osterholm MT, et al. Transmission of Ebola viruses: what we know and what we do not know. MBio. 2015;6(2):e00137.

29. Alfson KJ, Avena LE, Worwa G, Carrion R, Griffiths A. Development of a lethal intranasal exposure model of ebola virus in the cynomolgus macaque. Viruses. 2017;9(11):E319.

30. Wong G, et al. Ebola virus transmission in guinea pigs. J Virol. 2015; 89(2):1314-1323.

31. Mire CE, et al. Oral and conjunctival exposure of nonhuman primates to low doses of Ebola Makona virus. J Infect Dis. 2016;214(suppl 3):S263-S267.

32. Shacklett BL. Can the new humanized mouse model give HIV research a boost. PLoS Med. 2008;5(1):e13.

33. Valbuena G, Halliday H, Borisevich V, Goez Y, Rockx B. A human lung xenograft mouse model of Nipah virus infection. PLoS Pathog. 2014;10(4):e1004063.

34. Rodríguez E, et al. Humanized mice reproduce acute and persistent human adenovirus infection. J Infect Dis. 2017;215(1):70-79.

35. Jaiswal S, et al. Enhanced humoral and HLA-A2-restricted dengue virus-specific T-cell responses in humanized BLT NSG mice. Immunology. 2012;136(3):334-343.

36. Jangalwe S, Shultz LD, Mathew A, Brehm MA. Improved B cell development in humanized NOD-scid IL2R $\gamma^{\text {null }}$ mice transgenically expressing human stem cell factor, granulocyte-macrophage colony-stimulating factor and interleukin-3. Immun Inflamm Dis. 2016;4(4):427-440.

37. Shultz LD, Brehm MA, Garcia-Martinez JV, Greiner DL. Humanized mice for immune system investigation: progress, promise and challenges. Nat Rev Immunol. 2012;12(11):786-798.

38. Ellebedy AH, et al. Defining antigen-specific plasmablast and memory B cell subsets in human blood after viral infection or vaccination. Nat Immunol. 2016;17(10):1226-1234.

39. Davis CW, et al. Longitudinal analysis of the human B cell response to ebola virus infection. Cell. 2019;177(6):1566-1582.e17.

40. McElroy AK, et al. Kinetic analysis of biomarkers in a cohort of US patients with Ebola virus disease. Clin Infect Dis. 2016;63(4):460-467.

41. Groseth A, et al. The Ebola virus glycoprotein contributes to but is not sufficient for virulence in vivo. PLoS Pathog. 2012;8(8):e1002847.

42. Leung DW, et al. Structural and functional characterization of Reston Ebola virus VP35 interferon inhibitory domain. $J M o l$ Biol. 2010;399(3):347-357.

43. Groseth A, Ströher U, Theriault S, Feldmann H. Molecular characterization of an isolate from the 1989/90 epizootic of Ebola virus Reston among macaques imported into the United States. Virus Res. 2002;87(2):155-163.

44. Spengler JR, et al. Severity of disease in humanized mice infected with Ebola virus or Reston virus is associated with magnitude 
of early viral replication in liver. J Infect Dis. 2017;217(1):58-63.

45. Ryabchikova EI, Kolesnikova LV, Luchko SV. An analysis of features of pathogenesis in two animal models of Ebola virus infection. J Infect Dis. 1999;179(suppl 1):S199-S202.

46. Bowen ET, Platt GS, Simpson DI, McArdell LB, Raymond RT. Ebola haemorrhagic fever: experimental infection of monkeys. Trans R Soc Trop Med Hyg. 1978;72(2):188-191. 